Mine the Gap

The coming net-zero carbon revolution will entail a lot more mining.

Geologists need to be able make the case for this, at home and abroad, says Christopher Brough

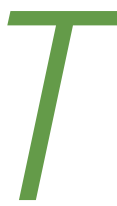

he industrial revolution paved the way for huge advances in the standard of living across the world. Globally, we now face a different challenge: the need to reach net zero carbon by 2050 to avert or minimise the worst effects of climate change.

The switch to net zero carbon will require wholesale transfer of energy production from carbon sources to renewable sources (solar, wind, wave, geothermal). It will also entail a revolution in the automotive sector as all vehicles, from private to public to haulage, become powered by lithium-ion (Li-ion) battery technology.

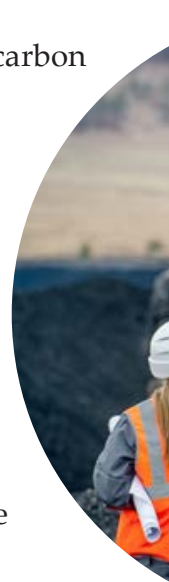
110

Mining will be crucial to meeting these challenges, as we source the material for the new energy mix and Li-ion batteries. Geologists will need to make the case for an industry which currently has a negative reputation amongst the public, particularly with respect to environmental issues, and is often only reported in terms of its capacity for exploitation and damage.

\section{Recyclability gap}

Renewable energy sources and Li-ion batteries are more material intensive than their carbon counterparts, which entails a huge increase in material demand. Estimations vary, from an increasing demand of hundreds of percent to thousands for several critical metals such as copper, lithium, graphite and cobalt. For some elements, this could mean the amount we need to mine over the next 25 years will be equivalent to the sum total extracted over the course of human history.

It remains to be seen how much of this can be supplied by improved recycling and a circular economy, but there is no version of the future that will not involve substantially upscaling the extraction of the materials we need.

\section{Skills gap}

Whilst I was undertaking further education my then supervisor was responsible for running the

exploration and mining course;
consistently one of the most popular to attend as students saw the practical benefit and job opportunities involved. University courses like these are going to become increasingly important. The skillsets required to start a mine are diverse, from exploration for the raw material to obtaining a social license to operate and the requisite stakeholder engagement. From mineralogists to engineers to hydrologists, our universities are going to need to train up a next generation capable of practically meeting the extractive demands of the net zero target.

\section{Transport gap}

Currently, the supply of many of these elements requires a complex globally linked chain totalling tens of thousands of kilometres back and forth. Reaching the net zero target will require shortening these chains where practicably possible and therefore bringing domestic supply chains and domestic mines back online.

One obviously can't build a mine without a resource to extract, but many European countries, including the UK, still have world class mining districts within their international borders. Part of their extraction will require geologists prepared to make the positive case to general media and the wider public that mining not only exists for the general good of society, but is absolutely integral to reaching our net zero target.

Christopher Brough is a Senior Consultant Mineralogist at Petrolab Ltd specialising in the application of petrology and mineralogy to the minerals and mining industry. (chris@petrolab.co.uk)

\section{SOAPBOX CALLING!}

Soapbox is open to contributions from all Fellows. You can always write a letter to the Editor, of course, but perhaps you feel you need more space?

If you can write it entertainingly in 500 words, the Editor would like to hear from you. Email your piece, and a self-portrait, to sarah.day@geolsoc.org.uk. Copy can only be accepted electronically. No diagrams, tables or other illustrations please.

Pictures should be of print quality - please take photographs on the largest setting on your camera, with a plain background.

Precedence will always be given to more topical contributions.

Any one contributor may not appear more often than once per volume (once every 12 months).

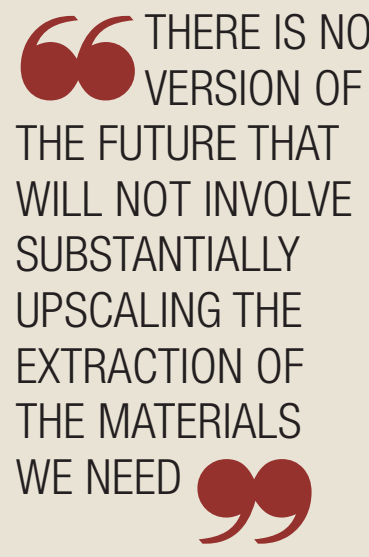

CHRISTOPHER BROUGH 